An international panel of six experts has reviewed the available worldwide data from clinical trials and prospective studies of PDE5-inhibitor therapy for ED. They identified the achievement and maintenance of maximal erection hardness as a key determinant of patients' overall satisfaction with treatment, as well as sexual satisfaction, selfesteem and confidence. The panel found that the Erection Hardness Score (EHS, a recently validated 4-point scoring system-unpublished to date) showed particular promise for assessing erection hardness. An EHS3 erection (hard enough for penetration but not completely hard) was not associated with sexual satisfaction, whereas an EHS4 erection (completely hard and fully rigid) was; furthermore, men whose most frequent erection type changed from EHS3 to EHS4 with therapy reported considerably improved sexual and psychological outcomes.

The panel concluded that the primary goal of PDE5-inhibitor therapy for men with ED should be achievement of full erectile potential. Quantitative assessments of erection hardness should be obtained before initiation of PDE5-inhibitor therapy and at each follow-up visit.

Original article Mulhall J et al. (2007) Erectile dysfunction: monitoring response to treatment in clinical practicerecommendations of an international study panel. J Sex Med 4: $448-464$

\section{Testis-derived transcript gene variants and prostate cancer risk in black men}

Recent studies suggest that the testisderived transcript gene TES is a candidate tumor suppressor gene for prostate cancer at chromosome 7q31.

To investigate this theory, Liu and colleagues evaluated the effects of seven tagging single nucleotide polymorphisms (SNPs) that comprehensively captured the common genetic variants in TES on aggressive prostate cancer in a case-control study. They recruited a total of 506 patients with aggressive prostate cancer, together with an equal number of age, institute and ethnicity-matched controls, and used a logistic regression model to evaluate the association between SNPs/multimarker haplotypes and prostate cancer. No statistically significant associations were seen between any variants and more aggressive disease when all study subjects and white men only were considered. However, three variants were inversely associated with disease severity in black men. These included two intronic SNPs (rs2402056, rs1004109) and one SNP close to the 3 ' untranslated region (rs4730721) with ORs of 0.57 (95\% Cl 0.36-0.90, under an additive mode of inheritance), 0.57 (95\% Cl 0.360.91 , under an additive mode of inheritance) and 0.45 (95\% Cl 0.21-0.98, under a dominant mode of inheritance), respectively.

While recognizing the limitations of the study, including the relatively small number of black men, the authors suggest that variants in TES, or in nearby genes, may be associated with prostate cancer in black men.

Original article Liu X et al. (2007) Association of testis derived transcript gene variants and prostate cancer risk. J Urol 177: 894-898

\section{Transurethral needle ablation of the prostate has poor long-term efficacy}

Transurethral needle ablation of the prostate (TUNA) was heralded as a minimally invasive alternative to transurethral resection of the prostate (TURP), but is now marketed as an alternative to pharmacotherapy. TUNA has short-term efficacy comparable to TURP in the treatment of lower urinary tract symptoms caused by benign prostatic hyperplasia, but long-term data are scanty. Rosario and colleagues, therefore, conducted a single-center, prospective study to evaluate long-term outcomes and cost-effectiveness of TUNA in men scheduled to undergo TURP after their obstructive symptoms failed to respond to $\alpha$-blockade.

Follow-up data were available for 70 of 71 consecutive men, aged $57.2-83.9$ years, who underwent TUNA. Patients' symptoms and urologic parameters were evaluated before TUNA and regularly during follow-up (range 102120 months). Adverse effects of TUNA were mild (hematuria, urinary-tract infection, inflammation) and resolved with treatment. Despite good 1-year results for TUNA, treatment efficacy declined with time. Only $17 \%$ of the cohort showed a sustained benefit throughout follow-up; symptoms recurred in the remainder (median time to recurrence 20 months). Men 